\title{
Granulomatous peritonitis caused by iatrogenic spillage of ovarian dermoid cystectomy: a case report and literature review
}

\author{
Hyo-Eun Kim, MD', Minji Seo, MD², Jae Young Kwack, MD², Yong-Soon Kwon, MD, PhD² \\ Department of Obstetrics and Gynecology, ${ }^{1}$ Ulsan University Hospital, University of Ulsan College of Medicine, Ulsan, ${ }^{2}$ Nowon Eulji Medical Center, \\ Eulji University, Seoul, Korea
}

\begin{abstract}
A 39-year-old nulliparous woman experienced continuous mild fever and abdominal pain since undergoing laparoscopic ovarian dermoid cystectomy 3 months previously in a local hospital. Abdominal computed tomography revealed diffuse heterogeneous fat infiltrations with numerous micronodules in the greater and lesser omentum, combined with ascites with thickening of the parietal peritoneum. The patient underwent exploratory laparoscopy, which included partial pelvic peritonectomy, excision of granulomas, and adhesiolysis with massive irrigation. The patient was treated successfully with laparoscopic surgery and all reproductive structures were spared without operative complications. To avoid peritonitis, complete removal of cyst contents and massive irrigation should be performed during ovarian dermoid cystectomy. Conservative surgical treatment may be a good choice for treating granulomatous peritonitis induced by iatrogenic rupture.
\end{abstract}

Keywords: latrogenic disease; Teratoma, ovarian; Peritonitis

\section{Introduction}

Ovarian dermoid cyst (ODC) is one of the most common benign ovarian neoplasms [1]. Ovarian cystectomy or oophorectomy is usually performed to treat associated pain symptoms to exclude malignancy and avoid potential complications such as torsion and spontaneous rupture. Laparoscopic ovarian cystectomy has become a routine procedure and has replaced traditional laparotomic approaches [2]. However, despite the advantages of laparoscopic treatment, there is an increased risk for intraperitoneal spillage of cyst contents that can potentially result in chemical peritonitis. Prolonged chemical peritonitis rarely results in the formation of granuloma in the peritoneum, otherwise known as granulomatous peritonitis (GP). To our knowledge, there has been only one reported case of prolonged GP, which was treated with repeated laparotomic surgeries [3]. The case described herein would be the second of iatrogenic GP due to spillage of ODC contents. However, it is the first to describe successful treatment of iatrogenic GP using conservative laparoscopic surgery.

\section{Case report}

A 39-year-old nulliparous woman visited the department of infection with symptoms of daily night fever and abdominal pain. She had a history of laparoscopic ovarian cystectomy, performed 3 months previously in a local hospital, due to an ODC. She also complained of other gastrointestinal symptoms including nausea, dyspepsia, intermittent constipation, and abdominal discomfort; however, she did not experience pelvic pain. Physical examination revealed mild tenderness

Received: 2019.10.05. Revised: 2019.11.12. Accepted: 2019.12.18. Corresponding author: Jae Young Kwack, MD

Department of Obstetrics and Gynecology, Nowon Eulji Medical Center, Eulji University, 68 Hangeulbiseok-ro, Nowon-gu, Seoul 01830, Korea

E-mail: podogazi@naver.com

https://orcid.org/0000-0001-6685-5894

Articles published in Obstet Gynecol Sci are open-access, distributed under the terms of the Creative Commons Attribution Non-Commercial License (http://creativecommons. org/licenses/by-nc/3.0/) which permits unrestricted non-commercial use, distribution, and reproduction in any medium, provided the original work is properly cited.

Copyright $\odot 2020$ Korean Society of Obstetrics and Gynecology 


\section{Obstetrics \& Gynecology Science}

Vol. 63, No. 4, 2020

noted throughout the abdomen. Laboratory investigations revealed increased levels of inflammatory markers, including the tumor marker cancer antigen 125 (CA-125), which was elevated to $189.8 \mathrm{U} / \mathrm{mL}$.

Abdominal computed tomography (CT) revealed diffuse heterogeneous, smudged, fat infiltrations with numerous micronodules in the greater and lesser omentum, along with ascites with thickening of the parietal peritoneum, and there was tubular enhancing granulation tissue in the right lower quadrant abdominal wall (Fig. 1A). The differential diagnosis included tuberculous peritonitis, malignant peritonitis, and GP, which in all cases involve elevated levels of CA-125. Consequently, the patient was referred to the department of general surgery and gynecology for simultaneous evaluation of the peritoneal and pelvic cavities, including biopsies of the nodules revealed on abdominal $\mathrm{CT}$.

The first laparoscopic surgery was performed in the depart- ment of general surgery. The operation revealed the presence of multiple nodular lesions, 1-3 mm in size, in the peritoneum and omentum. Laparoscopic findings revealed pelvic adhesion and approximately $500 \mathrm{~mL}$ of serous ascites (Fig. 1B). In addition to the peritoneum and omentum excision biopsies, ascites fluid was sent for cytological examination.

The patient experienced persistent fever, even after the first laparoscopic surgery, and was transferred to the department of gynecology for exploration of the pelvic cavity and subsequent gynecological surgical management. The second laparoscopic surgery was performed 2 weeks after the first operation. Operative findings included severe adhesions in the omentum, bowel to the peritoneum, uterus, and tube to ovary, with multiple granulomatous nodules in the lesser and greater omentum (Fig. 1C). Peritoneal and pelvic adhesiolysis was performed, in which prominent granulomas were removed, and the peritoneal and pelvic cavities were copi-
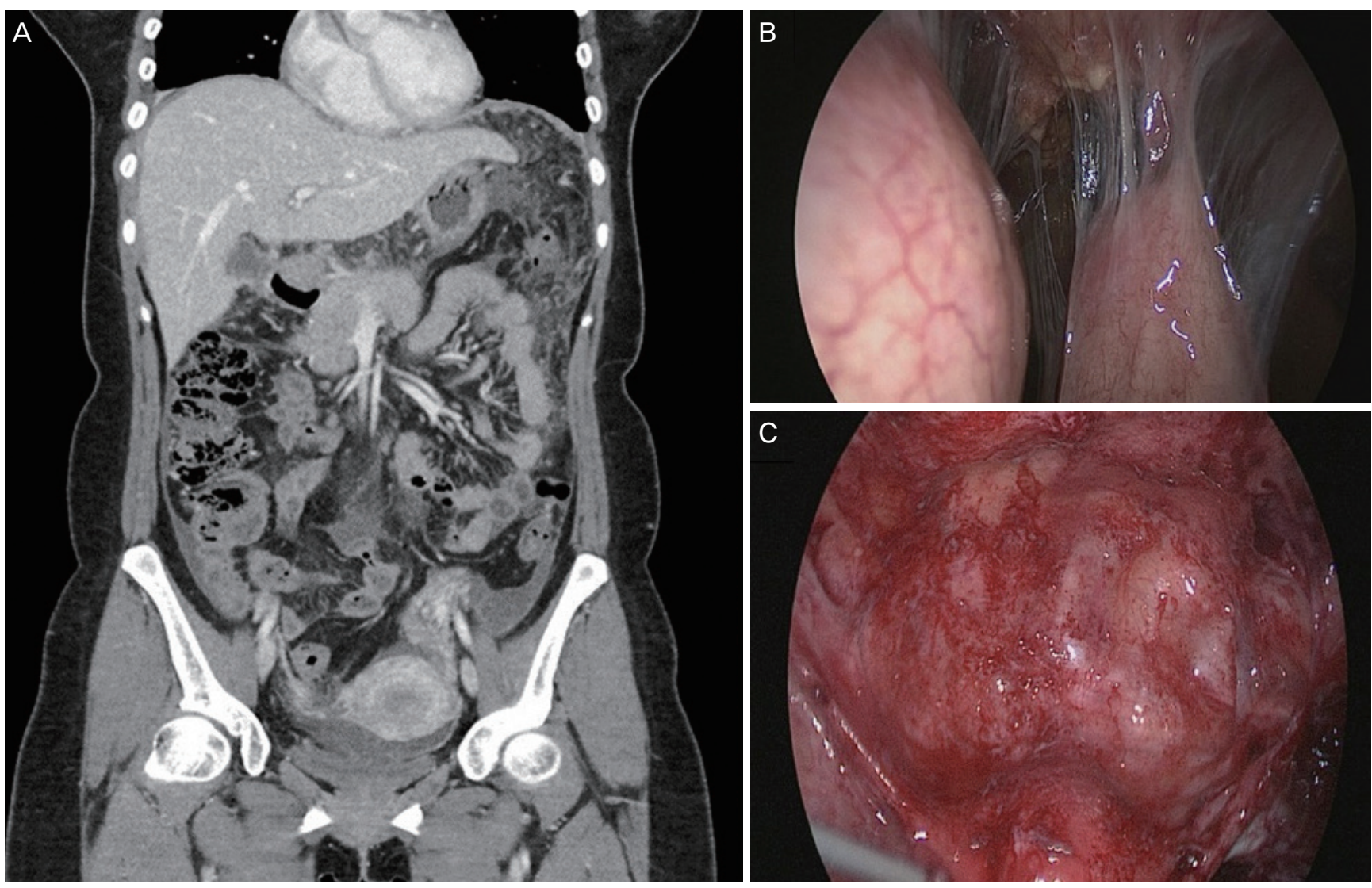

Fig. 1. Preoperative abdominopelvic computed tomography (CT) findings and intraoperative findings of granulomatous peritonitis. (A) Preoperative CT revealing diffuse moderate heterogeneous, smudged fat infiltrations with numerous micronodules in the greater and lesser omentum, combined with diffuse mild ascites with diffuse thickening of the parietal peritoneum, with tubular enhancing granulation tissue in the right lower quadrant abdominal wall. (B) Laparoscopic view revealing profuse sebaceous material in the entire pelvic cavity. (C) Laparoscopic view revealing multiple nodular lesions in the greater and lesser omentum. 


\section{Obstetrics \& Gynecology Science}

Hyo-Eun Kim, et al. Peritonitis after surgery of ovarian teratoma

ously irrigated. Frozen biopsy of the omental tissue revealed granulomatous inflammatory processes consistent with foreign-body reaction. However, no spillage of ODC materials trapped within the operative field was suspected.

Laboratory investigations revealed no evidence of malignancy or signs of tuberculosis in the ascites. The surgical specimens exhibited multifocal fibrotic changes with inflammatory cell infiltration (Fig. 2A), foreign-body type granulomas encompassing hair-like foreign materials (Fig. 2B and C), multinucleated giant cells within the granulomas (Fig. 2C), and non-caseating granulomas with chronic inflammatory cells (Fig. 2D). Biopsies of prominent nodules collected from the right pelvis and right para-colic gutter demonstrated a microscopic appearance similar to the surgical specimens. Interestingly, any suspected spillage of ODC contents was not observed either in laparoscopic exploration or in the irrigated ascites fluids. However, microscopic examination revealed hair-like materials in the resected granulomas.

The patient was started on a soft diet on postoperative day 3 and experienced significantly decreased gastrointestinal symptoms. The level of C-reactive protein increased to 7.77 $\mathrm{mg} / \mathrm{dL}$ on postoperative day 1 but returned to the normal range $(0.98 \mathrm{mg} / \mathrm{dL}) 1$ week later. The patient was discharged on postoperative day 4 of the second laparoscopic surgery. She remained free of symptoms at the 3-month follow-up, with no additional postoperative management.

\section{Discussion}

ODC is one of the most common benign ovarian neoplasms present during childbearing years [1]. Such cysts are usually benign teratomas that often contain diverse types of tissue including hair, teeth, thyroid parenchyma, and cystic components. Ovarian cystectomy is used to treat pain and avoid complications such as torsion and spontaneous rupture. Spontaneous rupture and rupture during cystectomy can lead to chemical peritonitis, as observed in the present case.

Laparoscopy is regarded to be a safe and efficient method for the diagnosis and treatment of ODCs by proficient laparoscopic surgeons. This procedure offers many advantages including less postoperative pain, lower risk for wound complication, earlier ambulation, and more rapid convalescence. The risk for adhesion formation is also reduced using this procedure. However, in the case of an ODC, the laparoscopic approach could result in chemical peritonitis caused by spilled contents of a ruptured dermoid cyst. Gynecological laparoscopy is generally performed with the patient in the Trendelenburg position, and there can be difficulty in suctioning contents after rupture, which can be hidden in the upper abdominal space [4]. If spillage of ODC contents is suspected, irrigation and changing patient position to the reverse Trendelenburg will help spilled contents flow downward from the upper abdomen.

Leakage of sebaceous material and hair follicles from the intraperitoneal rupture of mature cystic teratoma causes an aseptic inflammatory peritoneal reaction, specifically, chemical peritonitis. Although we could not find materials that induced GP on gross inspection in the second operation, microscopic examination revealed hair-like materials, from which we inferred the possibility of a small amount of spillage of ODC contents during laparoscopic ovarian cystectomy.
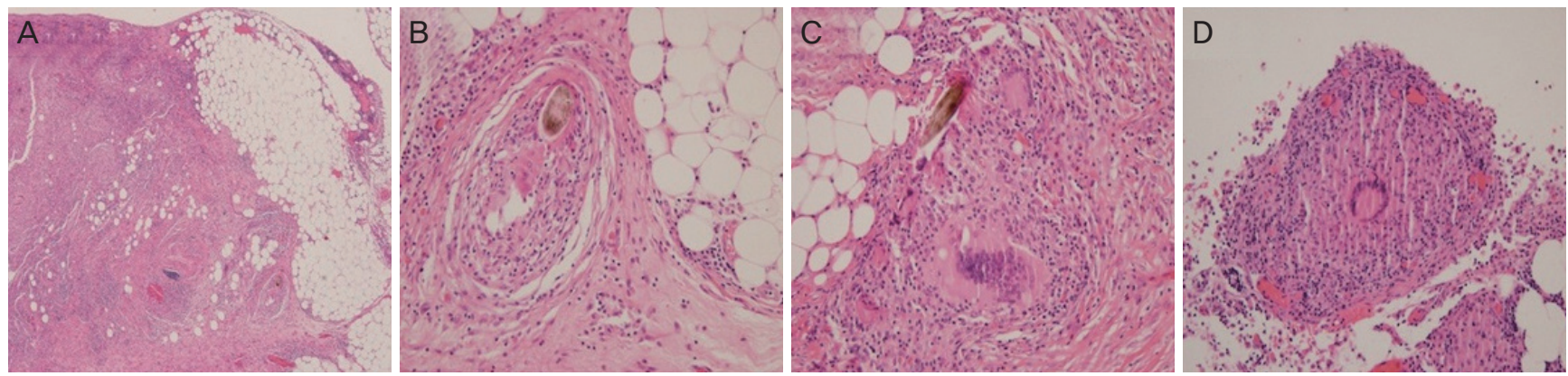

Fig. 2. Histological findings of peritoneal nodules. (A) Chronic inflammation and fibrosis of the omentum (hematoxylin and eosin [H\&E] staining, original magnification $\times 40$ ). (B) Hair follicle-like structure in the omentum (H\&E staining, original magnification $\times 200)$. (C) Damaged hair follicle-like structure with multinucleated giant cells (H\&E staining, original magnification $\times 200)$. (D) Foreign-body type granuloma (H\&E staining, original magnification $\times 200)$. 


\section{Obstetrics \& Gynecology Science}

Vol. 63, No. 4, 2020

Prolonged chemical peritonitis leads to granuloma formation in the peritoneum [4]. Chronic GP, however, is one of the catastrophic complications caused by intraoperative leaking of dermoid cyst contents. Moreover, the condition often appears along with severe adhesions, and ascites that resemble tuberculosis peritonitis or malignancy [5].

The occurrence of GP is very rare. Shawki et al. [6] reviewed 496 laparoscopic dermoid cystectomies in which spillage occurred in 324 (65.3\%) cases. Despite the high incidence of spillage, however, chronic GP developed in only 1 (0.2\%) patient. There are a few reports describing chronic GP due to spontaneous rupture of ODC $[7,8]$. To manage chronic GP due to spontaneous dermoid cyst rupture, hysterectomy with salpingo-oophorectomy or omentectomy using a laparotomy approach have been performed due to a clinical pattern that mimics advanced ovarian malignancy $[7,8]$.

To the best of our knowledge, the first case of iatrogenic GP caused by the spillage of ODC contents during laparoscopic surgery was reported by Huss et al. [3]. They treated the patient with 2 laparotomies: one trans-rectal and the other via xipho-pubic access.

The signs and symptoms of GP may be subtle and marginal in the early period of chemical peritonitis; however, the patient would complain of progressive abdominal distention, lower abdominal pain, and gastrointestinal disturbances such as anorexia, nausea, vomiting, and diarrhea [7].

Chronic GP usually exhibits unusual radiological findings such as ascites and omental infiltration and thickening of parietal peritoneum on CT scan. These findings may be confused with the peritoneal spread of advanced malignancy and tuberculosis [5]. Thus, ascites fluid analysis includes acidfast bacillus culture and staining, and/or cytology to rule out the presence of other malignancies; in addition, bacterial or fungal culture tests should also be evaluated. Significant elevation in serum CA-125 levels occurs in patients with tuberculous peritonitis and aseptic GP [9].

In the present case, conservative laparoscopic surgerysparing the uterus and both ovaries by only removing the prominent inflammatory tissues and copious lavage- - proved to be sufficient treatment for prolonged chemical peritonitis induced by dermoid cyst spillage. Granulomas encompassing foreign materials are relatively large and the surface mucosa is contagious and should be removed. If a patient experiences persistent gastrointestinal or pelvic symptoms and has suspicious findings of aggravated peritonitis on ultrasonog- raphy, repeated laparoscopic surgeries should be performed. Ruptured teratomas with peritonitis may be difficult to discriminate from malignancies [10]. In case of malignancy, thorough rinsing can reduce the possibility of direct seeding of tumor cells. As such, massive irrigation is essential when malignant tumor cannot be absolutely excluded [11]. In addition, copious saline lavage has been shown to decrease the risk for adhesion and inflammation [12]. Another technical point to prevent GP is careful dissection of the cyst and use of an endoscopic retrieval bag to remove cysts via a port site to prevent spillage [13].

In conclusion, surgeons should perform the technique in a less traumatic manner and be cautious about cystic rupture to prevent spillage. If, however, spillage occurs, complete removal of cyst contents and massive irrigation should be considered key measures to avoid peritonitis. It is apparent that an early conservative surgical approach with laparoscopy, involving removal of peritoneal granuloma and copious lavage, can result in successful treatment of advanced chemical peritonitis.

\section{Conflict of interest}

No potential conflict of interest relevant to this article was reported.

\section{Ethical approval}

This study was approved by Institutional Review Board of UIsan University Hospital (approval number 2018-03-035).

\section{Patient consent}

Written informed consent was obtained from each participant.

\section{References}

1. Comerci JT Jr, Licciardi F, Bergh PA, Gregori C, Breen JL. Mature cystic teratoma: a clinicopathologic evaluation of 517 cases and review of the literature. Obstet Gynecol 


\section{Obstetrics \& Gynecology Science}

Hyo-Eun Kim, et al. Peritonitis after surgery of ovarian teratoma

1994;84:22-8.

2. Shamshirsaz AA, Shamshirsaz AA, Vibhakar JL, Broadwell C, Van Voorhis BJ. Laparoscopic management of chemical peritonitis caused by dermoid cyst spillage. JSLS 2011;15:403-5.

3. Huss M, Lafay-Pillet MC, Lecuru F, Ruscillo MM, Chevalier JM, Vildé $F$, et al. Granulomatous peritonitis after laparoscopic surgery of an ovarian dermoid cyst. Diagnosis, management, prevention, a case report. J Gynecol Obstet Biol Reprod (Paris) 1996;25:365-72.

4. Clément $D$, Barranger $E$, Benchimol Y, Uzan S. Chemical peritonitis: a rare complication of an iatrogenic ovarian dermoid cyst rupture. Surg Endosc 2003;17:658.

5. Kaya M, Kaplan MA, Isikdogan A, Celik Y. Differentiation of tuberculous peritonitis from peritonitis carcinomatosa without surgical intervention. Saudi J Gastroenterol 2011;17:312-7.

6. Shawki O, Ramadan A, Askalany A, Bahnassi A. Laparoscopic management of ovarian dermoid cysts: potential fear of dermoid spill, myths and facts. Gynecol Surg 2007;4:255-60.

7. Phupong V, Sueblinvong T, Triratanachat S. Ovarian teratoma with diffused peritoneal reactions mimicking advanced ovarian malignancy. Arch Gynecol Obstet 2004;270:189-91.
8. Suprasert P, Khunamornpong S, Siriaunkgul S, Phongnarisorn $C$, Siriaree $S$. Ruptured mature cystic teratomas mimicking advanced stage ovarian cancer: a report of 2 cases study. J Med Assoc Thai 2004;87:1522-5.

9. Mas MR, Cömert B, Sağlamkaya U, Yamanel L, Kuzhan O, Ateşkan $U$, et al. CA-125; a new marker for diagnosis and follow-up of patients with tuberculous peritonitis. Dig Liver Dis 2000;32:595-7.

10. Stuart GC, Smith JP. Ruptured benign cystic teratomas mimicking gynecologic malignancy. Gynecol Oncol 1983;16:139-43.

11. Royal College of Obstetricians and Gynaecologists (UK). Management of adnexal masses in premenopausal women. Green-top guidelines No. 62. London: Royal College of Obstetricians and Gynaecologists; 2011.

12. Fielder EP, Guzick DS, Guido R, Kanbour-Shakir A, Krasnow JS. Adhesion formation from release of dermoid contents in the peritoneal cavity and effect of copious lavage: a prospective, randomized, blinded, controlled study in a rabbit model. Fertil Steril 1996;65:852-9.

13. Kondo W, Bourdel N, Cotte B, Tran X, Botchorishvili R, Jardon $K$, et al. Does prevention of intraperitoneal spillage when removing a dermoid cyst prevent granulomatous peritonitis? BJOG 2010;117:1027-30. 